\title{
Bilateral Adductor Palsy: A Rare Complication during Routine Surgery
}

\author{
Vidya Bhushan Rangappa, Athira Ramakrishnan, AM Balasubramanya, Raja Ram
}

\begin{abstract}
Recurrent laryngeal nerve (RLN) palsy occurring following thyroidectomy is a known complication. The first thing that comes to the surgeons, mind in a case of bilateral recurrent laryngeal nerve palsy is tracheostomy to secure the airway. But here we present a very interesting and unusual case of bilateral adductor palsy, post total thyroidectomy. We also highlight on the fact that not always the surgeon is to be blamed and there are certain subtle other factors to be aware of.
\end{abstract}

Keywords: Vocal fold, Palsy, Adductor palsy, Thyroidectomy, Recurrent laryngeal nerve.

How to cite this article: Rangappa VB, Ramakrishnan A, Balasubramanya AM, Ram R. Bilateral Adductor Palsy: A Rare Complication during Routine Surgery. World J Endoc Surg 2013;5(3):76-78.

\section{Source of support $\mathrm{Nil}$}

\section{Conflict of interest: None}

\section{INTRODUCTION}

Recurrent laryngeal nerve (RLN) palsy occurring following thyroidectomy is a known complication. In most instances, it is assumed that the cause of the nerve injury is surgical. However, in spite of meticulous surgery and preservation of thenerves, rarely RL N palsy can still occur. We encountered one such case where the patient devel oped bilateral adductor vocal cord palsy following total thyroidectomy. We felt the cause for the pal sy is not related to the surgery. We present the events that occurred in our patient.

\section{CASE REPORT}

A 32-year-old female was referred to the outpatient department of surgical oncology for the surgical management of her toxic multinodular goiter. The patient gave a history of a neck swelling since 1 year, associated with weight loss and pal pitations for 6 months. Following diagnosis of the toxic multinodular goiter, she had been started on antithyroid medication. Examination revealed a multinodular goiter involving both lobes measuring $6 \times 5 \times 4 \mathrm{~cm}$. Patient had no voice symptoms and an office laryngoscopy showed bilateral mobile vocal cords. Total thyroidectomy under general anesthesia was planned. Patient was induced with propofol, intubated with a cuffed flexometal lic endotracheal tube (size $7 \mathrm{~mm}$ ) and maintained with isoflurane, nitrous oxide and oxygen. During the intraoperative period, the entire course of recurrent laryngeal nerve was identified and preserved. No electrocautery was used in the vicinity of the nerve. All the major blood vessels were ligated only after identifying the recurrent laryngeal nerve. No anatomical variation of the recurrent laryngeal nerve was noted. Duration of surgery was 3 hours and 20 minutes and the patient was positioned in the traditionally described extended position throughout the surgery. General anesthesia was reversed with neostigmine and patient was extubated smoothly. The immediate postoperative period was uneventful. Three hours postoperatively, the patient was noted to have a breathy voice and devel oped cough on taking oral feeds. She had no breathing difficulty or stridor. Postoperative laryngoscopic evaluation revealed bilateral fixed vocal cords with a wide phonatory gap of around $8 \mathrm{~mm}$. There was no evidence of edema or signs of trauma. The patient was started on nasogastric tube feeds and was referred to out speech therapist for vocal cord adduction exercises. The histopathological examination of the resected thyroid gland was suggestive of nodular colloid goiter. The patient was started on replacement dose of thyroxine and advised to review after 2 weeks. A fter 14 days, office laryngoscopic examination revealed flickering movements of the vocal cords with a persistent phonatory gap. Though the voice appeared unchanged to us, the patient felt there was an improvement in her voice. She was tolerating soft food orally but had persistence of aspiration to liquid feeds and hence was advised to continue tube feeds. Six weeks post surgery the patient, visibly revealed, came to the clinic with a normal voice and was able to eat and drink normally. Repeat office endoscopy showed bilateral normal and mobile vocal cords with complete adduction. The nasogastric tube was removed and the patient was advised to follow-up regularly.

\section{DISCUSSION}

One of the main complication of thyroid surgery is injury to the RLN. The surgical cause of the injury and the management of the complication have been described extensively. Patients with bilateral RLN palsy following thyroid surgery usually have their cords in the paramedian position and develop stridor. Tracheostomy is usually performed and the further management depends on the recovery of the nerves. It is assumed (and quiet rightly so) that the injury is surgical as the nerves are in the field of surgery. Rarely, the nerves can be affected due to causes 
not related to the surgery. $Y$ amashita et al ${ }^{1}$ in 1965 suggested that the bilateral RLN palsy they encountered was possibly related to the cuff overexpansion. Cadaver studies have been undertaken to provide an explanation to this phenomenon. Ellis et $\mathrm{al}^{2}$ found that the RL $N$ divides into an anterior and posterior branch below the superior rim of the cricoid cartilage. The anterior branch passes medial to the thyroid cartilage to supply the lateral cricoarytenoid and thyroarytenoid muscles. They suggest that a highly placed cuff can cause pressure neuropraxia of the RL N. In the study by $\mathrm{Cavo}^{3}$ et al, they found that the anterior branch of the RLN could be compressed between the expanded cuff of the endotracheal tube and the overlying thyroid cartilage. This should lead to paralysis of the muscles supplied by the anterior branch and lead to adductor palsy. A nother contributing factor could be an increase in cuff pressure during surgery due to diffusion of gases through the semipermeable membrane of the cuff. This may be a contributing factor, especially for prolonged surgeries. Extended position of the neck, commonly used in head and neck surgeries, can cause the cuff to ride up toward the larynx and cause pressure neuropraxia of the anterior branch of the RLN .

The anterior branch of the RLN supplies the adductor muscles of the vocal cord. When injured, it will result in an abducted vocal cord. Clinically, this would manifest with a hoarse voice and aspiration, but should not compromise the airway. This was the picture seen in our patient and has been described earlier in patients not undergoing head and neck surgery. As the airway is patent, tracheostomy is not required in these patients. The aspiration symptoms are very distressing and tube feeds should be initiated to maintain the nutrition. Speech and swallowing therapy will be useful for these patients. Recovery of the function of the vocal cords will eventually occur provided there is no permanent damage due to ischemia. Knowledge of this phenomenon is useful as the patient can be counseled well and be avoided of the morbidity and stress of a tracheostomy.

K nowledge of relations of recurrent laryngeal nerve at the level of thyroid gland is essential to avoid nerve damage during surgery of the gland. The recurrent laryngeal nerve enters the larynx, below or through the inferior fibers of the cricopharyngeal part of the inferior constrictor muscle. In tracing the recurrent laryngeal nerve superiorly, the first branch (posterior) that arises, innervates the posterior cricoarytenoid muscle. The next branch (anterior) to come off the recurrent laryngeal nerve, is the nerve to the interarytenoid muscle. ${ }^{3}$ The terminal branches of the recurrent laryngeal nerve innervates the lateral cricoarytenoid and thyroarytenoid muscles. The most frequent sites of damage during surgery are: (1) the cross point with the inferior thyroid artery, (2) the segment related to the gland capsule, (3) the segment which passes through the gland ligament, (4) the point of penetration into the larynx, bel ow or between the fibers of cricopharyngeal part of inferior constrictor, (5) the recurrent laryngeal nerve injury could result from transection, clamping, stretching, electrothermal injury, ligature entrapment or ischemia, but the actual causes of nerve injury are not well understood, especially in those nerves in which visual inspection during the operation confirmed nerve integrity. ${ }^{5}$ These mechanisms discussed above unfortunately cannot explain bilateral symmetrical vocal cord paralysis as seen in our patient. Paralysis of recurrent laryngeal nerve has been described as a rare complication of endotracheal intubation in cases where the patient had been free from laryngeal symptoms before the procedure, and where a cuffed intubation tube has been used al though nerve was not surgically manipulated and postoperatively no signs of intubation trauma were observed. ${ }^{2}$ The reason for vocal cord paralysis appearing in connection with anesthesia has generally been attributed to nerve compression caused by the cuff of intubation tube. ${ }^{2}$ The anterior branch of recurrent laryngeal nerve passes medial to the lamina of the thyroid cartilage to supply the lateral cricoarytenoid and thyroarytenoid muscle. With the palsy of the anterior branch of the nerve, only the posterior cricoarytenoid, interarytenoid and cricothyroid muscles are left functioning. ${ }^{3}$ In this area, the anterior branch of this nerve is vulnerable to compression between the expanded cuff and overlying thyroid cartilage on the superoanterior border of posterior cricoarytenoid muscle, which is about 6 to $10 \mathrm{~mm}$ below the posterior third of true vocal cord. ${ }^{4} \mathrm{As}$ the anterior branch of the recurrent laryngeal nerve exclusively innervates the adductors, bilateral paralysis of this branch results in cords being in the intermediate or more abducted position. ${ }^{1}$

L aryngoscopic examination of our patient postoperatively showed a similar picture with both vocal cords being in abducted position with a phonatory gap of approximately $8 \mathrm{~mm}$. It has been observed that the gases used during anesthesia can become infused into the cuff consequently increasing cuff pressure. ${ }^{2}$ Probably, a gas pressure gradient across the semipermeable membrane of endotracheal tube cuff is the source of cuff expansion causing pressure on intralaryngeal portion of recurrent laryngeal nerve and causing neuropraxia. ${ }^{4}$ Traditionally during neck surgeries, patient is positioned with neck hyperextended. Hyperextension of the neck during intubation or an operative procedure can cause stretching and paralysis of the vagus nerve which is anchored by the recurrentlaryngeal nervein the mediastinum. ${ }^{2}$ This position can also result in migration of the cuff proximally to just below the vocal cords aggravating 
pressure injury. ${ }^{1}$ In the differential diagnosis of the cord paralysis after an intubation, the possibility of arytenoids dislocation must also be kept in mind. ${ }^{2} \mathrm{H}$ owever in our case, this possibility was ruled out by a direct laryngoscopic examination and palpation of arytenoids.

\section{CONCLUSION}

Vocal fold paralysis following an endotracheal intubation recovers completely or nearly completely in more than half the cases. ${ }^{2} \mathrm{~A}$ Iso in surgical paresis of the recurrent laryngeal nerve, whether reversible or permanent, the best result is achieved by commencing voice training as early as possible $e^{2}$ as demonstrated by our case. In addition to meticulous surgical technique, cuff pressure monitoring and avoiding prolonged hyperextension should also be ensured to avoid probable nerve injury. These types of palsies usually totally improve in approximately 6 months and they require symptomatic management as noted. If cord movements do not recover for more than 6 months, medialization of the vocal fold can be considered.

\section{REFERENCES}

1. $Y$ amashita $T$, Harada $Y$, U eda $N$, et al. Recurrent laryngeal nerve paralysis associated with endotracheal anesthesia. J Otorhinolaryngol Soc J pn 1965;68:1452-1459.

2. Ellis PD, Pallister WK. Recurrent laryngeal nerve palsy and endotracheal intubation. A nesthesiology 1976;45:448-449.
3. Cavo J ohn W J r. True vocal cord paralysis following intubation. Laryngoscope 1985;95:1352-1358.

4. Cox RH, W elborn SG. Vocal cord paralysis after endotracheal anesthesia. South M ed J 1981;74:1258-1259.

5. Feng-Y u C, I-Chen Lu, Wen-Rei K, Ka-Wo L, Ning-Chia C, Che-W ei $W$. The mechanism of recurrent laryngeal nerve injury during thyroid surgery - the application of intraoperative neuromonitoring. O riginal Research A rticle Surgery 2008 J une; 143(6):743-749.

\section{ABOUT THE AUTHORS}

\section{Vidya Bhushan Rangappa (Corresponding Author)}

Postgraduate, D epartment of ENT, Head and Neck Surgery, St. J ohn's M edical College Hospital, B engaluru, Karnataka, India, Phone: 22065735, e-mail: drbhushanhns@gmail.com

\section{Athira Ramakrishnan}

Senior Resident, Department of ENT, Head and Neck Surgery, St. J ohn's Medical College Hospital, B engaluru, Karnataka, India

\section{AM Balasubramanya}

Professor, Department of ENT, Head and Neck Surgery, St. John's M edical College Hospital, B engaluru, Karnataka, India

\section{Raja Ram}

Assistant Professor, Department of Surgical Oncology, St. John's M edical College Hospital, B engaluru, Karnataka, India 